\title{
A Historical Survey of Sir Karl Popper's Contribution to Quantum Mechanics
}

\author{
William M. Shields
}

Worcester Polytechnic Institute, Worcester, Massachusetts, United States. E-mail: highc.king@verizon.net

Editors: Ion C. Baianu, Christoph Lehner, Debajyoti Gangopadhyay \& Danko Georgiev

Article history: Submitted on May 28, 2012; Accepted on July 28, 2012; Published on November 15, 2012.

ir Karl Popper (1902-1994), though not trained as a physicist and embarrassed early in his career by a physics error pointed out by Einstein and Bohr, ultimately made substantial contributions to the interpretation of quantum mechanics. As was often the case, Popper initially formulated his position by criticizing the views of others in this case Niels Bohr and Werner Heisenberg. Underlying Popper's criticism was his belief that, first, the Copenhagen interpretation of quantum mechanics abandoned scientific realism and second, the assertion that quantum theory was complete (an assertion rejected by Einstein among others) amounted to an unfalsifiable claim. Popper insisted that the most basic predictions of quantum mechanics should continue to be tested, with an eye towards falsification rather than mere adding of decimal places to confirmatory experiments. His persistent attacks on the Copenhagen interpretation were aimed not at the uncertainty principle itself and the formalism from which it was derived, but at the acceptance by physicists of an unclear epistemology and ontology that left critical questions unanswered.

Quanta 2012; 1: 1-12.

(c) (1) This is an open access article distributed under the terms of the Creative Commons Attribution License CC-BY-3.0, which permits unrestricted use, distribution, and reproduction in any medium, provided the original author and source are credited.

\section{Popper in the physics journals}

Sir Karl Popper, by any measure one of the preeminent philosophers of the twentieth century, died in 1994 at the age of 92. He was productive to the end, publishing in the year of his death a criticism of Kuhn's incommensurability of paradigms [1]. That debate continues over his many and profound philosophical ideas and opinions is hardly surprising, almost two decades after his death. The proliferation of book-length biographies and scholarly philosophical articles is testimony to Popper's standing as a philosopher [2-5]. Major conferences are also regularly held on the thought of Karl Popper [6,7].

It may come as a surprise, however, that beginning in the year 2000, Popper's name appears prominently in no less than a dozen papers in the journals of theoretical physics, in the majority of cases in the paper's title [8-20]. Several of these papers report the results of Popper's experiment carried out by physicists at the University of Maryland in 1999 [13-17]. Coincidentally, the final section of a recent text on the Einstein-Podolsky-Rosen (EPR) paradox in physics deals with this same experiment, proposed by Popper in the early 1980's [21. Sec. 5.4.3]. How is it that physicists in the new millennium are invoking Karl Popper's name, conducting experiments suggested by him, and arguing over the meaning of the results?

To suggest answers to this question, I review below Popper's fifty years of contributions to the interpretation of quantum mechanics. For reasons that will become clear 
in later sections of the paper, I trace a line of thought experiments that Popper proposed to test his own views against the views of the majority of theoretical physicists who created quantum mechanics. In the final section of the paper, I offer a possible explanation for why Popper's passionately-held opinions continue to attract the attention of physicists, and are worthy of that attention.

\section{2 'Logik Der Forschung' and Einstein's refutation}

The most exciting and fundamental discoveries of quantum mechanics were made while Popper was in college and graduate school. Though his dissertation was not in physics, he had studied the sciences and mathematics and was qualified to teach them on the secondary level. The thesis, completed in 1928, was titled On the Problem of Method in the Psychology of Thinking and it had more to do with the methodology of science than psychology. Popper characterized it as a "hasty last minute affair" [22, p. 78]. By the time he began work in earnest on Logik der Forschung in the early 1930's, Popper had educated himself on the new quantum mechanics, by then becoming accepted as a major advance in atomic physics.

Consistent with his interest in the logic and methodology of science, Popper focused his study on the philosophical underpinnings of the new theory. He was especially interested in the disputes that had arisen over how to interpret physically the mathematical formalism of the theory. Popper explains:

At the time (1930) when ... I began writing my book, modern physics was in turmoil. Quantum mechanics had been created by Werner Heisenberg in 1925, but it was several more years before outsiders - including professional physicists - realized that a major breakthrough had been achieved. And from the very beginning there was dissension and confusion. The two greatest physicists, Einstein and Bohr, perhaps the two greatest thinkers of the twentieth century, disagreed with one another. [22, pp. 90-91]

Lacking doctoral-level knowledge of physics, Popper struggled to grasp the new theory:

I was working on my own from books and from articles; the only physicist with whom I sometimes talked about my difficulties was my friend Franz Urbach. I tried to understand the theory and he had doubts whether it was understandable - at least by ordinary mortals. [22, p. 91].
Eventually Popper came to appreciate the core of the disagreement within physics and was able to sort out in his mind the various positions of Einstein, Bohr, Heisenberg, Schrödinger, and Born. By the time Logik Der Forschung was well underway, Popper felt qualified to address quantum theory in the book.

Never one to tackle a subject halfway, Popper devoted all of Chapter IX of Logik Der Forschung to Some Observations on Quantum Theory [23]. In the English translation, it runs to 35 densely-argued pages wherein Popper sets out the views he was to maintain, with some modifications, for the rest of his life [24]. He makes his purpose clear in the introductory section:

What follows here might be described, perhaps, as an inquiry into the foundations of quantum theory. In this, I shall avoid all mathematical arguments and, with one single exception, all mathematical formulae. This is possible because I shall not question the correctness of the system of mathematical formulae of quantum theory. I shall only be concerned with the logical consequences of its physical interpretation which is due to [Max] Born. [24, p. 216]

Thereafter Popper lays out his criticism of what is commonly called the Copenhagen Interpretation of quantum theory (largely the work of Bohr) and the position of Heisenberg that the uncertainty relations must be viewed subjectively, as a "limitation of our knowledge" of physical systems [24, p. 220]. In the same passage, Popper notes that Moritz Schlick of the Vienna Circle had expressed strong support for Heisenberg's views (Heisenberg's explication of his own position can be found in [25]).

Popper devotes the next several sections to advocacy of a statistical interpretation of the uncertainty relations. He argues that, contra Heisenberg, it does indeed make sense to attribute well-defined positions and momenta to individual particles. Experimental results showing wavelike behavior of particles (as in slit experiments) can be explained as "statistical scatter relations." The scattering behavior is calculated using the mathematical machinery of quantum theory, but it does not imply anything about limitations on knowledge or an actual lack of a well-defined position and momentum at any moment in time [24, p. 225].

With this interpretation in hand, Popper wonders aloud whether anything has in fact been gained. His conclusion emphasizes his aim, which is in essence to succeed where Einstein had failed in his arguments with Bohr:

The statistical elements of quantum theory must be inter-subjectively testable in the same way 
as any other statements of physics. And my simple analysis preserves not only the possibility of spatio-temporal descriptions, but also the objective character of physics. [24, p. 234]

Events that took place two years after the publication suggested to Popper that he should have ended the chapter there. But he did not. In the next sections of the chapter, Popper describes an imaginary experiment (Gedankenexperiment) "which shows, in full agreement with quantum theory, that the precise measurements in question are possible" [24, p. 243]. I will omit details of the experimental design here - suffice to say that it had the same idea as later versions, but was flawed in several key respects.

These flaws were made apparent to Popper at a scientific conference held in Copenhagen in 1936. Following the conference, Popper was invited by Bohr at the urging of Victor Weiskopf, a leading theoretician, to stay on a few days to discuss quantum mechanics. Popper was already feeling uneasy about his Gedankenexperiment, which had been questioned by Einstein. After discussions with Bohr, Popper accepted that the experiment did not show what he intended, and he left Copenhagen quite upset over losing the argument [22, pp. 92-93]. While he did not stop thinking about quantum mechanics, he "remained for years greatly discouraged ... I could not get over my mistaken thought experiment" [22, p. 94]. But following consultations with physicist Arthur March in the late 1940's, Popper returned to the problems of quantum mechanics with "something like renewed courage" [22, p. 94]. He began to revise and clarify his ideas while working on a set of appendices for the English version of Logik Der Forschung and the long-delayed Postscript. (The Postscript actually appears several years before the English edition of the book with its new appendices. Here I consider the appendices first in order to conclude the discussion of Logik Der Forschung.)

Appendix xi of the English version of Logik Der Forschung offers some general thoughts on the use of imaginary experiments in physics (see also [26, pp. 240265]) before moving to a lengthy discussion of the Einstein-Podolsky-Rosen (EPR) experiment and Bohr's interpretation of it, which Popper predictably rejects. In Appendix xii, Popper bites the bullet and reprints in full Einstein's letter to him of 1935, in which Einstein describes the flaws in the Gedankenexperiment proposed in Logik Der Forschung. Popper admits that Einstein's letter "briefly and decisively disposes of my imaginary experiment in section 77" of Logik Der Forschung [24, p. 457]. By this time, however, Popper had already proposed a new experiment in the Postscript to Logik Der Forschung, and it is to that work that I now turn.

\section{3 'Postscript to Logik Der Forschung': thoughts on Gedankenexperiments}

Popper's Postscript evolved by the mid-1950s into a massive work that outsized the work it was a postscript to, so large that it had to be published in three volumes [27--29]. Popper's determination to have an impact on quantum theory is evidenced by his devoting the entire third volume to the subject. If Quantum Theory and the Schism in Physics [29] shows nothing else, it is proof that in the twenty years since his embarrassment before Bohr, he had devoted immense effort to mastering quantum mechanics. The book covers a wide range of quantum theoretical controversies and points of view, with a continual mixing of physical, mathematical, and philosophical ideas.

To explore this work fully is beyond my present scope. (This work appeared first in 1956, but again in a new (and more easily obtainable) version in 1982. The 1982 version, which in many ways represents Popper's final statement of views on quantum theory, opens with a 35page Preface written in 1982, followed by a 62-page chapter written as a paper in 1966! The 1956 volume entitled Schism actually begins at page 97 of the 1982 edition. So the 1982 edition actually reads backwards from 1982 to 1956. In this section I confine the discussion to the 1956 material.) For the moment I wish to focus on Popper's revisiting of the idea of an experimentum crucis. As to the imaginary experiment proposed in Logik Der Forschung, Popper states bluntly that it was "invalid, and I wish to withdraw it" [29, p. 98]. In Chapter III, he discusses first the relatively simple experiment of collimated particles aimed at a small slit in a barrier. Classical mechanics would say that the particles will travel straight through, while quantum theory demands that if the slit is small enough, a scattering effect will be achieved. This scattering effect is due, according to Heisenberg, to the confinement of the wave packet representing the particle to a distance $\Delta x$ (the width of the slit), causing according to the uncertainty relations a corresponding uncertainty in the momentum $\Delta p$, where $\Delta x \Delta p \geq \hbar$ [25, pp. 23-24]. But where Heisenberg (and Bohr) interpreted this result (which is easily observable) as amounting to a denial of a scattered particle's "particality," so to speak, so that retrodictive calculation of the particle's path is essentially meaningless, Popper argues that one can conclude no such thing. In his view, each scattered particle had a real path and had a well-defined position and momentum at all times. It was simply scattered by the slit: no epistemological conclusions can be drawn beyond that [29, pp. 144-147]. Popper then proceeds to examine the arguments based on the celebrated Gedankenexperiment proposed by 
Einstein, Podolsky and Rosen, forever thereafter known as the EPR experiment [30].

After a discussion of the conflicting points of view of Einstein and Bohr - with interjections on why he agrees with Einstein - Popper does not mince words on what he believes is at stake:

Reasonableness was the point at issue. The question is not whether by a subtle and highly scholastic argument we may continue to uphold an untenable position. The question is whether we should think critically and rationally in physics, or defensively and apologetically. [29, p. 150]

It is fairly apparent who Popper believes is doing the critical thinking and who is being defensive and apologetic.

Finally, Popper turns to an experiment analyzed (using classical wave theory) by Thomas Young in the early 1800's and, according to Popper, "discussed again and again by Bohr" [29, p. 151]. This is a two-slit experiment, in which the quantum scattering induced by one slit is then "projected" onto another barrier with two slits in it, neither of which aligns with the slit in the first barrier. What will then be observed, again assuming that all the slits are small enough to engage quantum phenomena, is an interference pattern just as if the whole experiment were conducted with macroscopic waves on a pond. But because we are using discrete particles, and each particle can go through only one of the two secondary slits, it appears that the two slits cooperate in producing the interference pattern. Indeed, closing one or the other of the paired slits changes the final pattern.

Popper uses this experiment as an opportunity to apply his propensity interpretation of probability calculations. Where Bohr would say that the result in each case (all slits open, two open, etc.) can be explained only be recourse to the complementary notions of wave and particle along with considering the active role of the observer, Popper argues that "it is the whole experimental arrangement which determines the propensities." By 'propensities' here, Popper refers to his interpretation of probability, which differs from the more commonly-held view that probability is statistical in nature. In Popper's view, probability is more akin to a field of force than it is to a mathematically-calculated frequency. It therefore acquires a 'reality' which can have physical effects: the probability fields can interact and interfere with one another. This approach, which Popper modified and refined over the years, responds to Einstein's dictum that 'God does not play dice', and tends to restore some measure of determinacy to physics that the standard versions of quantum theory deny. (Popper's approach to probability, especially as it is used in physics, is discussed at various points throughout [29]; see also [31, pp. 59-60] [3, pp. 109-112]. A recent paper compares Popper's view on propensities with those originally suggested by the American philosopher Charles Sanders Peirce [32].) I mention the Young experiment, especially the aspect of closing one of the paired slits, because it appears to be a precursor in Popper's mind of a modified two-slit experiment he will propose and defend from 1981 to 1987 . This new experimental proposal is the subject of the next section. I have found no evidence that Popper's 1956 efforts in the Postscript generated any interest in the physics community. Most likely, the Postscript was read by few physicists.

\section{Gedankenexperiment refinements, 1981-1987}

Popper's solitary efforts to offer a different view of quantum phenomena acquire a different status in the early 1980's. By this time, he had acquired colleagues in the theoretical physics community, one of whom, French physicist Jean-Pierre Vigier, had an international reputation. This collaboration led to the publication of a paper in a widely-read English-language journal, Physics Letters, in December of 1981 [33]. Italian physicist Augusto Garuccio, K. Popper and J.-P. Vigier (GPV) jointly proposed an experiment involving the interference of laser beams. The intent of the paper is clearly stated in the opening paragraph and rather obviously drafted by Popper:

The present letter develops a gedanken experiment which leads to conflicting testable predictions of the Copenhagen ... and causal statistical ... interpretations of quantum theory. [33, p. 397]

It is not clear why the term "gedanken" is used here, since the experiment was clearly within the range of 1981 technology. Perhaps the term is used only to make clear that they had not built the apparatus and showed that it could perform as predicted. The GPV experimental design was based on work done by two other physicists, L. Mandel and R. L. Pfleegor, in the late 1960's, and modified an experiment first proposed by Garuccio and Vigier in 1980. Popper and his co-authors acknowledge helpful comments not only from Mandel, but also from John S. Bell, who derived the famous Bell's inequalities, and Alain Aspect, who was at that time (1981) conducting experiments of his own to test Bell's inequalities. The paper's concluding assertions include a comment on demarcation that must surely have been Popper's: 
As one knows this typical Copenhagen retroactive action (which has been used to justify parapsychological phenomena) raises trouble with energy conservation and implies rejection of Feynman's quantum propagator $D_{c}$. The authors feel with Einstein that: (I) the flow of time is a real, irreversible and one-dimensional phenomenon, (II) only positive energies move in the forward time direction, and (III) the apparent microscopic time reversibility of the quantum mechanical wave equations only reflects the particle/anti-particle mixture of Einstein and Feynman which leads to correct perturbation theory. [33, p. 400]

More or less coincident with the publication of this paper, Popper re-issued Quantum Theory and the Schism in Physics with a new Preface, On a Realistic and Commonsense Interpretation of Quantum Theory and a new first section, Quantum Mechanics Without 'The Observer' [29. pp. 1-96]. Towards the end of the Preface, Popper proposes "a simple thought experiment which may be regarded as an extension of the Einstein-Podolsky-Rosen argument." Oddly, this experiment (though in this case admittedly a thought experiment, as no apparatus is actually described) is not at all the same as in the paper with Vigier and Garuccio, though the underlying purpose is the same. This experiment involved the placing of an emission source between two slits. The source emits particles with equal and opposite momenta (Figure 1). The particles pass through the small slits and scatter according to the uncertainty relations. But what would happen, Popper asks (and here is where the centuries-old Young optics experiment may have played a role), to the observed scattering on one side if the slit on the other side is widened so that by itself it would cause no quantum scattering? Will the "knowledge" imparted by the confinement of the particle in the still-small slit induce scattering on the other side so that the observed pattern does not change? As Popper explains:

To sum up: if the Copenhagen interpretation is correct, then any increase in the precision of our mere knowledge of the position $q_{y}$ of the particles going to the right should increase their scatter; and this prediction should be testable. [29. p. 29]

The paper in Physics Letters drew immediate attention in the physics community. First to respond was French physicist O. Costa de Beauregard, who offered in May of 1982 a brief letter entitled Disagreement with Garuccio, Popper and Vigier [34]. De Beauregard had no apparent objections to the experimental design of GPV but argued that the results would be fully consistent with the standard interpretations of quantum theory. He admits, however, that his remarks "contain no objection against the tentative theory of Garuccio et al." Just a few weeks later, Mandel criticized GPV's experimental arrangement and offered a modification of the design that would in principle address his objections [35]. The next month, June 1982, Garuccio and Vigier entered another paper in Physics Letters, but in this case Popper's place as a co-author was taken by one of Garuccio's colleagues, V. Rapisarda [36]. That Popper remains involved is made clear by note 4 in this paper, which cites a "private communication" from Popper. These authors noted that the GPV paper had "provoked a complex and heated discussion" in the physics community. Their present purpose was to

...present an experimental programme and experimental set-up which clearly falls outside of the field of the above-mentioned objections to the preceding discussion [GPV] and suppresses, as far as possible, experimental difficulties. [36 p. 17]

At the end of the paper, the authors asserted that this experiment will "escape all preceding objections" and, if conducted, "would really constitute a crucial distinction between CIQM [the Copenhagen Interpretation] and reality."

There the matter lay until 1985. In that year, a paper by Anthony Sudbery, a mathematician at the University of York, appeared in Philosophy of Science [37]. Sudbery's critical stance can be discerned from his lengthy title: Popper's Variant of the EPR Experiment Does Not Test the Copenhagen Interpretation. Sudbery analyzed the version of the experiment Popper presented in the 1982 Preface to Quantum Theory and the Schism in Physics, and in fact made no mention at all of the 1982 papers in Physics Letters. Sudbery criticizes two features of the thought experiment:

The essential elements of [Popper's] deduction are: (i) the inverse relation between $\Delta y$ and $\Delta p_{y}$, the uncertainties in position and momentum; and (ii) the correlation between the positions of two particles that have interacted in the past. Neither of these is universally true, whatever interpretation of quantum mechanics is in question; each of them holds only in special circumstances. According to the Copenhagen interpretation, they do not hold simultaneously; hence Popper's deduction of the effect $E$ [increase in momentum spread] is not valid within this interpretation. [37, p. 472] 

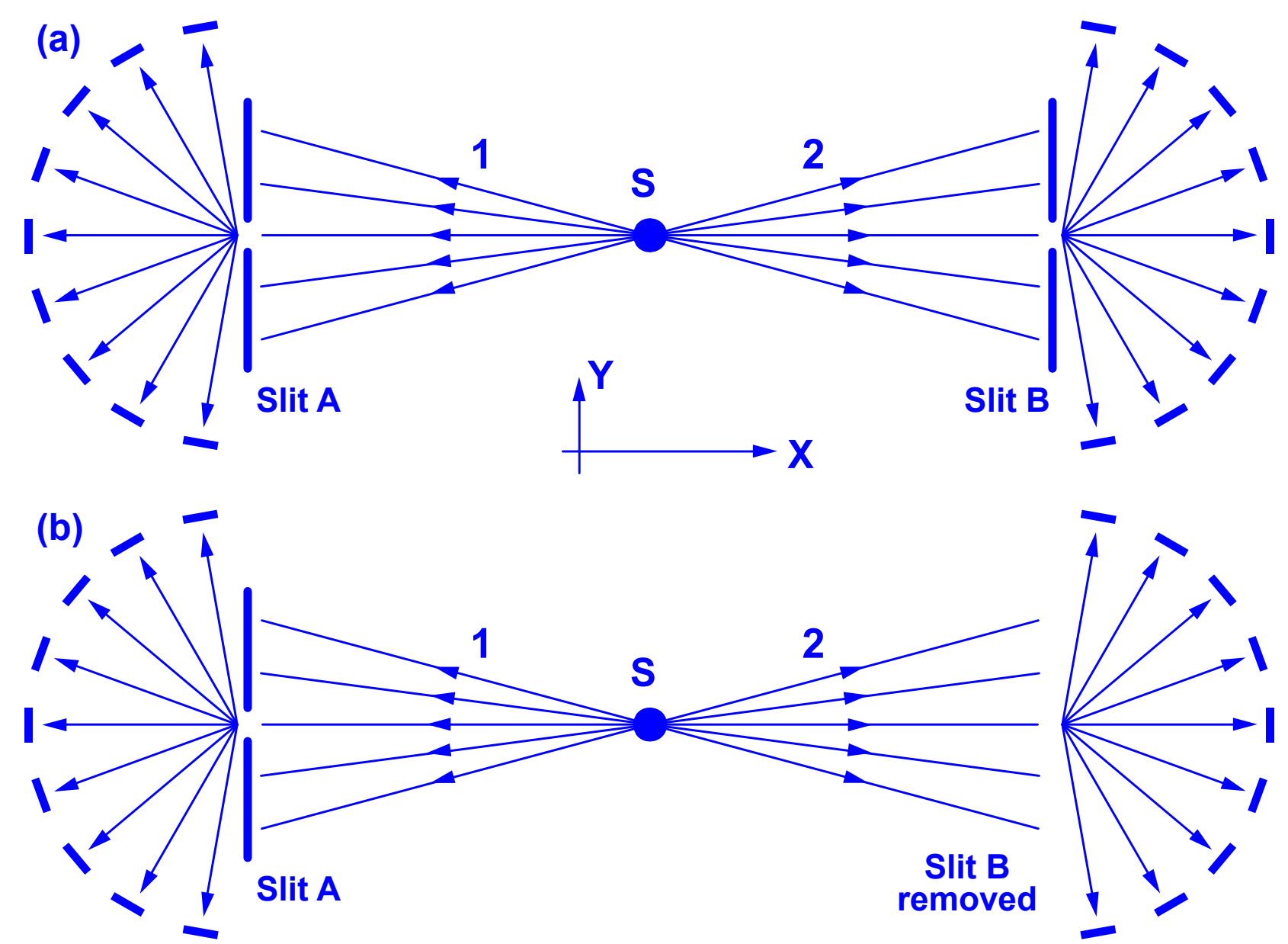

Figure 1: Schematic diagram of Popper's thought experiment. (a) When both slits A and B are present, the particles are expected to show scatter in momentum. (b) Popper believed that by removing slit B one could test the Copenhagen interpretation.

As to (i), Sudbery points out that the inverse relation (i.e., the Heisenberg relation) is not an equality but an inequality. Using the entangled wave function employed by Einstein, Podolsky and Rosen (the EPR wave function), application of the inequality leads to the possibility that the uncertainties in the positions and momenta of the entangled particles is in fact infinite. In such a case, Sudbery argues, "narrowing slit A has no effect on the range of momenta of the particles at either $\mathrm{A}$ or $\mathrm{B}$, since these ranges cannot be increased any further". On element (ii), Sudbery argues that the experimental apparatus used to measure the entangled particles after separation, by the Copenhagen interpretation, disturbs the initial correlations between positions and momenta. Hence the wave function being measured is not the EPR function describing the initial state, which Sudbery writes in the form:

$$
\psi\left(\mathbf{r}_{A}, \mathbf{r}_{B}\right)=\phi_{1}\left(x_{A}\right) \phi_{2}\left(x_{B}\right) \delta\left(y_{A}-y_{B}\right)
$$

where $\phi_{1}$ and $\phi_{2}$ are separated localized wave packets, but a different wave function in which the correlations are no longer present:

$$
\psi^{\prime}\left(\mathbf{r}_{A}, \mathbf{r}_{B}\right)=\phi_{1}\left(x_{A}\right) \phi_{2}\left(x_{B}\right)
$$

He summarizes as follows:

If the particles approach the slits in the EPR wave function, so that the observation of one particle gives information about the other, then the spread of the counters that register particles do not depend on the width of the slit. Conversely, if the experiment is arranged so that the spread of counters does depend on the width of the slit, the observation of one particle gives no information about the other. [37, p. 473]

Although some researchers have rejected Sudbery's logic and conclusions [38], in his recent paper, Qureshi [20] finds that Sudbery's argument on (i) above "is the only robust criticism of Popper's experiment".

That Popper's views were not universally rejected, however, is made apparent in the 1985 text Open Questions in Quantum Physics, based on the proceedings of a conference held in 1983 at the University of Bari, Italy [39]. Popper's contribution, Realism in Quantum Mechanics and a New Version of the EPR Experiment [40] appears first in the book, and is followed by a discussion among Popper, Vigier, and nine other physicists [39, pp. 26-32]. 
Popper's essay in this book represents, in my view, the culmination of his thinking on quantum theory and its relationship to scientific realism and human knowledge. In the opening paragraphs, he comments:

I am a realist, and I believe in the reality of matter, or energy, of particles, of fields of forces, of wavelike disturbances of these fields, and of propensity fields (de Broglie fields) ... and I suggest that quantum mechanics is misinterpreted when it is not interpreted realistically. I also suggest that quantum mechanics says nothing whatever about epistemology, about our knowledge and its limits, no more than Newtonian dynamics. [40, p. 4]

Contrast this view with that of Heisenberg, one of Popper's epistemological nemeses. In describing the meaning of the uncertainty relations as applied to the position and momentum of a free electron following a precise measurement of its velocity and no measurement at all of position:

Then the principle states that every subsequent observation of the position will alter the momentum by an unknown and indeterminable amount such that after carrying out the experiment our knowledge of the electronic motion is restricted by the uncertainty relation ... It is a matter of personal belief whether [backcalculation of] the past history of the electron can be ascribed any physical reality or not. [25. p. 20]

Bohr, the other principal target of Popper's criticism, argued to the same effect:

Indeed we have in each experimental arrangement suited for the study of proper quantum phenomena not merely to do with an ignorance of the value of certain physical quantities, but with the impossibility of defining these quantities in an unambiguous way. [41, p. 699]

A few pages into the essay, Popper characteristically joins the issue without hesitation; he has come a long way from the embarrassment of the Logik experiment:

[This leads to] the doctrine that the Heisenberg formulae $\Delta p_{x} \Delta q_{x} \geq \frac{h}{2 \pi}$ etc. are about limits to human knowledge or to the precision of possible measurements on particles. This Copenhagen thesis I deny. As a realist I assert that the formula is about the lower limits on the scatter of particles ... The particles themselves possess sharp positions and, at the same time, sharp momenta. [40, p. 4-5]
The discussion following Popper's essay was spirited and focused almost entirely on Popper's proposed experiment, which in this essay is the same as the experiment proposed in the Postscript, i.e., particles with correlated momentum states passing through slits. Some participants doubted it could be conducted, others argued over what the results (one way or the other) might mean. Gino Tarozzi, one of the conference organizers, was strongly in favor of conducting the experiment, commenting that in his view the experiment would be an effective test of "Einstein locality versus Heisenberg's indeterminacy relations" 39 . p. 30].

Popper's final contributions to the interpretation of quantum mechanics appeared in the pages of Letters to Nature in 1987 [42,43]. Two physicists at Essex University, M. J. Collett and R. Loudon, had taken the position that Popper's 1982 Postscript experiment "does not in fact provide a test" of the Copenhagen Interpretation [44, 45]. They argued that the source (say positronium) must be assigned a finite uncertainty in both position and momentum; calculation of this "source uncertainty effect" showed, in their view, that experiment was not a valid test of the Copenhagen interpretation. Popper, now 85 years old, replied to the letter a few months later. He began by thanking Collett and Loudon for "opening up a discussion of my 1982 proposal ... an experiment based upon Einstein, Podolsky and Rosen and a radical simplification of another proposal by myself," a reference to the ill-fated Logik experiment [42]. Popper answered the physicists' arguments point for point, and corrected their impression that his experiment was a test of quantum mechanics itself:

My experiment was never intended as a crucial experiment of quantum mechanics but only of its (subjectivist) Copenhagen interpretation (which they call "the standard interpretation"). [42]

Popper pointed out that there "exist several interpretations of the formalism" and provided a citation to one developed by Jon Dorling. (Indeed, work continues along the lines Popper advocated, i.e., a statistical interpretation of quantum mechanics and especially of the uncertainty relations. See, for example, [46], the abstract of which begins: "I attempt to develop further the statistical interpretation of quantum mechanics proposed by Einstein, developed by Popper, Ballentine, etc.”) Collett and Loudon's reply to this letter followed immediately after Popper's letter; they simply do not agree [45]. Popper insisted in characteristic fashion on having the last word. In a short note in Letters to Nature a month later, he first corrected a rather obvious error in the printing of his previous letter [43]. Then he took one last jab at Collett and Loudon's position: 
I would point out that in their original criticism they speak of a "fixed source", whereas in their new criticism they replace this by a "massive source." To my mind this means a change of the problem: they never explain why a (nonmassive positronium) source cannot be "fixed." [43]

Popper died in 1994, and thereafter, one might expect, his Gedankenexperiment would draw no further notice. Indeed, that was the case for more than a decade after 1987. But in 1999, Popper returned to the pages of the physics journals, and in a most surprising way.

\section{Shih and Kim, 1999, and aftermath}

In 1999, University of Maryland physicists Yanhua Shih and Yoon-Ho Kim reported the results of a realization of Popper's experiment [13-15]. Their experimental setup did not use Popper's point particle source (such as a decay of positronium) - it used entangled photons produced by a laser and refracted by lenses through slits (Figure 2). This arrangement avoided the "Sudbery problem" of the inability to eliminate uncertainty effects in the initial position of a particle. Shih and Kim point out that

... a point source is not a necessary requirement for Popper's experiment. What is needed is the position entanglement of a two-particle system, i.e., if the position of particle 1 is precisely known, the position of particle 2 is also $100 \%$ determined. [14, p. 466]

Their results, taken at face value, "show that there appears to be a violation of the uncertainty principle" [14, p. 463]. This would mean, from Popper's point of view, that the Copenhagen interpretation is in error.

But Shih and Kim do not take that position. Instead, they argue that it is impermissible to apply the uncertainty relations to each of the entangled-state photons separately. These photons are, in their view, represented by a "nonfactorizeable two-dimensional wave packet" such that " $\Delta y \Delta p_{y} \geq \hbar$ is not applicable to either photon 1 or photon 2 individually." They conclude:

Our experimental demonstration of Popper's thought experiment call (sic) our attention to the important message: the physics of an entangled two-particle system is inherently different from that of two individual particles. [14, $\mathrm{p}$. 470]
It is of interest that among the physicists whose assistance is acknowledged are none other than Jean-Pierre Vigier and Augusto Garuccio, Popper's 1981 collaborators [14. p. 470].

Shih and Kim's paper generated a cloudburst of responses, comments, criticisms, and suggestions for further work [8,-12, 16, 17, 47,-53]. The positions taken vary from Asher Peres's ungracious reference to "the absurdity of Popper's result" [49, p. 23] to Geoffrey Hunter's affirmation that

Popper and EPR made no error - they agreed with Bohr, Heisenberg and other proponents of the Copenhagen interpretation that quantum theory predicts an instantaneous action at a distance ... Popper and EPR's crucial point is that if such actions at a distance are not in fact observed (as in the Shih-Kim experiment), then quantum theory must be an incomplete (only statistical) theory of the physical world ... [8, p. 248]

Rainer Plaga suggested an improvement ("Extension Step 1") in the Shih-Kim experiment that addresses a "conceptual flaw" having to do with the role of the observer [11]. In Plaga's opinion

... it is of great importance to actually perform Popper's experiment with "Extension Step 1" ... Should an experimental realization of "Extension Step 1" show that no virtual diffraction occurs, the relation between "quantum mechanical state" and "observed reality" ... would be put into doubt. [11, p. 471]

A. J. Short agreed with Shih and Kim that their results do not suggest a violation of the uncertainty principle, but for different theoretical reasons [47,48], while Brazilian physicist G. Rigolin disagreed with Short and claimed to "invalidate" his analysis [50]. A Korean group suggested a realization of Popper's experiment using a "dual measurement scheme" to achieve a modern version of Heisenberg's microscope thought experiment [10]. From Spain comes theoretician Pedro Sancho's application of Feynman's path integral methods to the Shih-Kim results [12]. And from one of India's leading theoreticians, C. S. Unnikrishnan, no less than three papers (all published since 2000) on many aspects of EPR, Popper's experiment, and the Shih-Kim results [51-53]. In addition to this spate of papers, Popper's experiment is analyzed in a recent textbook by Alexander Afriat and Franco Selleri devoted entirely to the EPR paradox [21] (published just slightly before Shih and Kim announced their results in 1999). The authors, both long-time contributors in the field, point 
(a)

D1

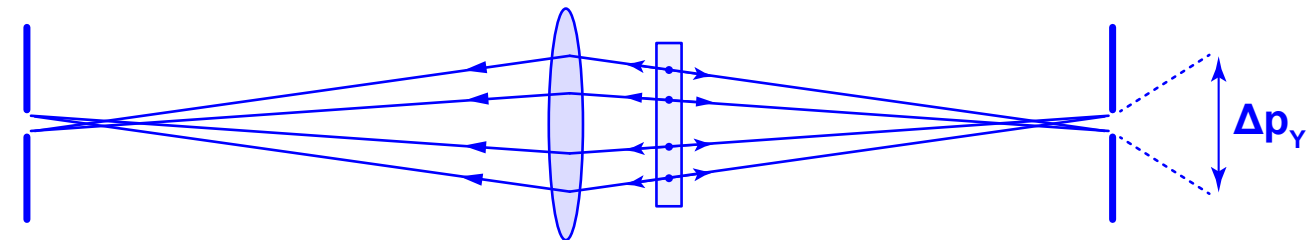

Slit B

Slit A

Lens BBO

Scan

Coincidence detection

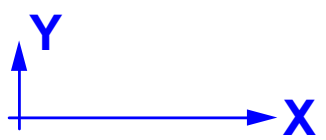

(b)

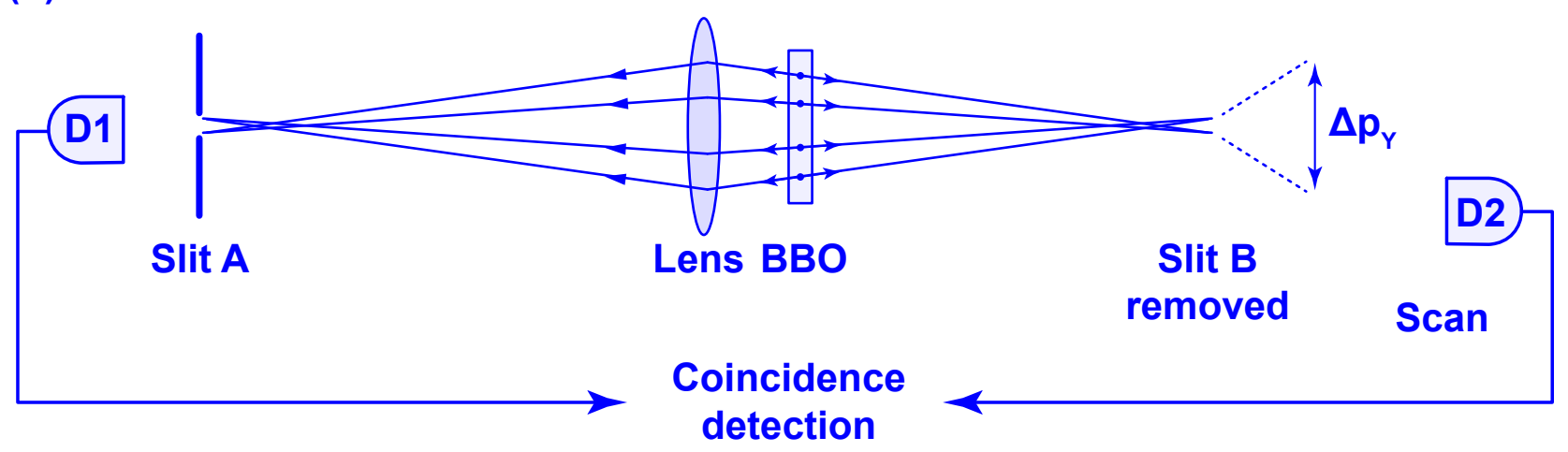

Figure 2: Modified version of Popper's experiment. An EPR photon pair is generated by spontaneous parametric downconversion in a barium borate $(B B O)$ crystal. A lens and a narrow slit A are placed in the path of photon 1 to provide the precise knowledge of its position on the $y$-axis and also determine the precise $y$-position of its twin, photon 2 , on screen $B$ due to a 'ghost image' effect. The distance between the lens and each of the slits is adjusted to $2 f$, where $f$ is the focal length of the lens. Two detectors D1 and D2 are used to scan in the $y$-directions for coincidence counts. (a) Slits A and B are adjusted both very narrowly. (b) Slit $A$ is kept very narrow and slit $B$ is removed.

out the initial momentum problem in Popper's original proposal, but also note that the experiment could in principle be conducted by the use of collinear particles or photons [21, pp. 238-242].

\section{Realism, uncertainty, knowledge}

In this final section, I wish to explore the question: what nerve did Popper strike in his persistent challenge to the Copenhagen interpretation? What motivates physicists to devote time and resources to attempt Popper's experiment and battle over the meaning of the results?

To Popper, the interpretation of quantum mechanics represented a demarcational issue, not between science and non-science, but between the physical reality of things and human knowledge of those things. Popper read Bohr and Heisenberg as suggesting that "mere knowledge" of things had an observable physical effect, and to Popper this was nearing something like belief in the paranormal.
He could not accept mixing ontology and epistemology in this way [54]. Things exist, and we can come to know them by conjectures and refutations - but what we know (or think we know) and what is are not causally connected. Ontology remains prior to epistemology.

But more than realism was at stake. Popper tells us in the opening pages of Schism that his "strongest reason for my own opposition to the Copenhagen interpretation lies in its claim to finality and completeness" [29, pp. 5-6]. This statement calls to mind the 1935 dispute between Einstein, Podolsky and Rosen on the one hand, and Niels Bohr on the other. The Einstein paper concludes:

While we have thus shown that the wave function does not provide a complete description of physical reality, we left open the question of whether or not such a description exists. [30, p. 780]

Bohr's responding paper certainly appears to lay a claim to finality and completeness: 
Such an argumentation [EPR], however, would hardly seem suited to affect the soundness of quantum-mechanical description, which is based on a coherent formalism covering automatically any procedure of measurement like that indicated. [41, p. 696]

To Popper, this argument must have seemed like a contest between the critical, questioning posture he developed in Logik Der Forschung, and a position bordering on pure empiricism: we can know no more than what we see, and what we can know limits what exists. Philosopher Michael Redhead (who both met and corresponded with Popper) explains that in Popper's view, "probabilities in physics cannot, in general, be epistemic. How could human ignorance produce genuine physical effects?" [55, p. 172]. Popper designed and promoted "his experiment" not so much to prove quantum mechanics wrong, as to restore the "conjectures and refutations" attitude in quantum theory. To Popper, a physicist arguing that his theory is final and complete was anathema.

Perhaps most of the physicists who are once again engaged in a debate over 'Popper's experiment' are not overly concerned, as he was, with the nature of valid scientific inquiry. That is not terribly important. What is important is that Popper continues, from beyond the grave, to prick the "standard interpretation" of quantum mechanics and in so doing unsettles the field enough to generate renewed debate. One can hardly improve on Redhead's appreciation of Popper's contribution:

Popper fought a lone battle against the Copenhagen interpretation at a time when anyone attempting to criticize orthodoxy was liable to be labeled at best an 'outsider' or at worst a crank. But Popper's carefully argued criticisms won the support of a number of admiring and influential physicists. He has done a great service to the philosophy of quantum mechanics by emphasizing the distinction between state preparation and measurement and trying to get a clearer understanding of the true significance of the uncertainty principle, but above all by spearheading the resistance to the dogmatic tranquilizing philosophy of the Copenhagenists. Because some detailed arguments are flawed, this does not mean that his overall influence has not been abundantly beneficial. [55, p. 176]

I believe that if Popper could once again weigh in on the debate - and weigh in he surely would! - he would demand above all else that the arguments continue, that more experiments be conducted, conjectures offered and refutations put forward. True, he would hope for a result that showed human knowledge has nothing whatever to do with the position and momentum of particles. Yet even if the result seemed to show otherwise, he would continue to look for ways to preserve scientific realism and objectivity. What he could not abide was any hint of smugness, a complacency that our knowledge is complete and critical inquiry is at an end.

\section{Acknowledgements}

I am grateful to Hanspeter Fetz of the London School of Economics for pointing out Redhead's work to me.

\section{References}

[1] Popper KR. The Myth of the Framework. London: Routledge, 1994.

[2] O’Hear A. Karl Popper. London: Routledge, 1980.

[3] Stokes G. Popper: Philosophy, Politics and Scientific Method. Cambridge: Polity Press, 1998.

[4] Jarvie I, Pralong S. Popper's Open Society After 50 Years. London: Routledge, 1999.

[5] Hacohen MH. Karl Popper - The Formative Years, 1902-1945: Politics and Philosophy in Interwar Vienna. Cambridge: Cambridge University Press, 2000.

[6] Alai M, Tarozzi G. Karl Popper Philosopher of Science: Proceedings of the Conference, Cesena, 2730/10/1994. Soveria Mannelli: Rubbettino, 2006.

[7] Amsterdamski S. Significance of Popper's Thought: Proceedings of the Conference Karl Popper: 19021994, March 10-12, 1995, Graduate School for Social Research, Warsaw. Amsterdam: Rodopi, 1996.

[8] Hunter G. Realism in the realized Popper's experiment. AIP Conference Proceedings 2002; 646 (1): 243-248. http://arxiv.org/abs/ quant-ph/0507011

[9] Muller FA. Refutability revamped: how quantum mechanics saves the phenomena. Erkenntnis 2003; 58 (2): 189-211. http://philsci-archive. pitt.edu/1368/

[10] Nha H, Lee J-H, Chang J-S, An K. Atomic-position localization via dual measurement. Physical Review A 2002; 65 (3): 033827. http://arxiv.org/ abs/quant-ph/0106053 
[11] Plaga R. An extension of "Popper's experiment" can test interpretations of quantum mechanics. Foundations of Physics Letters 2000; 13 (5): 461-476. http://arxiv.org/abs/quant-ph/0010030

[12] Sancho P. Popper's experiment revisited. Foundations of Physics 2002; 32 (5): 789-805. http: //dx.doi.org/10.1023/A:1016009127074

[13] Kim Y-H, Shih Y. Experimental realization of Popper's experiment: violation of the uncertainty principle? Foundations of Physics 1999; 29 (12): 1849-1861. http://arxiv.org/abs/quant-ph/ 9905039

[14] Shih Y, Kim Y-H. Experimental realization of Popper's experiment - violation of the uncertainty principle? Fortschritte der Physik 2000; 48 (5-7): 463-471. http://arxiv.org/abs/ quant-ph/9905039

[15] Shih Y, Kim Y-H. Quantum entanglement: from Popper's experiment to quantum eraser. Optics Communications 2000; 179 (1-6): 357-369. http://dx. doi.org/10.1016/s0030-4018(99)00716-6

[16] Shih Y. Quantum imaging, quantum lithography and the uncertainty principle. European Physical Journal D 2003; 22 (3): 485-493. http://dx. doi org/10.1140/epjd/e2003-00037-5

[17] Shih Y. Quantum imaging, quantum lithography and the uncertainty principle. Fortschritte der Physik 2003; 51 (4-5): 487-497. http://dx.doi.org/ $10.1002 /$ prop. 200310066

[18] Qureshi T. Popper's experiment, Copenhagen interpretation and nonlocality. International Journal of Quantum Information 2004; 2 (3): 407-418. http://arxiv.org/abs/quant-ph/0301123

[19] Qureshi T. Understanding Popper's experiment. American Journal of Physics 2005; 73 (6): 541-544. http://arxiv.org/abs/quant-ph/0405057

[20] Qureshi T. Analysis of Popper's experiment and its realization. Progress of Theoretical Physics 2012; 127 (4): 645-656. http://arxiv.org/ abs/quant-ph/0505158

[21] Afriat A, Selleri F. The Einstein, Podolsky, and Rosen Paradox: In Atomic, Nuclear, and Particle Physics. New York: Plenum Press, 1999.

[22] Popper KR. Unended Quest: An Intellectual Autobiography. New York: Routledge, 1982.
[23] Popper KR. Logik der Forschung. Wien: Springer, 1934.

[24] Popper KR. The Logic of Scientific Discovery. New York: Basic Books, 1959.

[25] Heisenberg W. The Physical Principles of the Quantum Theory. New York: Dover, 1949 (original edition published in 1930).

[26] Kuhn TS. The Essential Tension: Selected Studies in Scientific Tradition and Change. Chicago: Chicago University Press, 1977.

[27] Popper KR. Postscript to the Logic of Scientific Discovery. Vol. 1: Realism and the Aim of Science. Totowa, New Jersey: Rowman and Littlefield, 1983.

[28] Popper KR. Postscript to the Logic of Scientific Discovery. Vol. 2: The Open Universe: An Argument for Indeterminism. Totowa, New Jersey: Rowman and Littlefield, 1982.

[29] Popper KR. Postscript to the Logic of Scientific Discovery. Vol. 3: Quantum Theory and the Schism in Physics. Totowa, New Jersey: Rowman and Littlefield, 1982.

[30] Einstein A, Podolsky B, Rosen N. Can quantummechanical description of physical reality be considered complete? Physical Review 1935; 47 (10): 777-780. http://dx.doi.org/10.1103/ PhysRev.47.777

[31] Popper KR. Conjectures and Refutations: The Growth of Scientific Knowledge. New York: Routledge, 1963.

[32] Suárez M. Propensities and pragmatism, 2011. http://philsci-archive.pitt.edu/8957/

[33] Garuccio A, Popper KR, Vigier J-P. Possible direct physical detection of de Broglie waves. Physics Letters A 1981; 86 (8): 397-400. http://dx.doi. org/10.1016/0375-9601(81)90346-7

[34] Costa De Beauregard O. Disagreement with Garuccio, Popper and Vigier. Physics Letters A 1982; 89 (4): 171-172.http://dx.doi.org/10.1016/ 0375-9601(82)90200-6

[35] Mandel L. Tests of quantum mechanics based on interference of photons. Physics Letters A 1982; 89 (7): 325-326. http://dx.doi.org/10.1016/ 0375-9601(82) 90183-9 
[36] Garuccio A, Rapisarda V, Vigier J-P. New experimental set-up for the detection of de Broglie waves. Physics Letters A 1982; 90 (1-2): 17-19. http://dx.doi.org/10.1016/ 0375-9601(82)90038-X

[37] Sudbery A. Popper's variant of the EPR experiment does not test the Copenhagen interpretation. Philosophy of Science 1985; 52 (3): 470-476.

[38] Angelidis T. On some implications of the local theory $\mathrm{Th}(\mathrm{g})$ and of Popper's experiment. In: Gravitation and Cosmology: From the Hubble Radius to the Planck Scale, Amoroso R, Hunter G, Kafatos M, Vigier J-P (editors), Springer Netherlands, 2002, pp.525-536. http://dx.doi.org/ $10.1007 / 0-306-48052-2 \_56$

[39] Tarozzi G, van der Merwe A. Open Questions in Quantum Physics: Invited Papers on the Foundations of Microphysics. Boston: D. Reidel, 1985.

[40] Popper KR. Realism in quantum mechanics and a new version of the EPR experiment. In: Open Questions in Quantum Physics: Invited Papers on the Foundations of Microphysics, Tarozzi G, van der Merwe A (editors), Boston: D. Reidel, 1985, pp.3-25.

[41] Bohr N. Can quantum-mechanical description of physical reality be considered complete? Physical Review 1935; 48 (8): 696-702. http://dx.doi. org/10.1103/PhysRev.48.696

[42] Popper KR. Popper versus Copenhagen. Nature 1987; 328 (6132): 675. http://dx.doi.org/10 $1038 / 328675 \mathrm{a} 0$

[43] Popper KR. Correction needed. Nature 1987; 329 (6135): 112.http://dx.doi.org/10.1038/ 329112b0

[44] Collett MJ, Loudon R. Analysis of a proposed crucial test of quantum mechanics. Nature 1987; 326 (6114): 671-672. http://dx.doi.org/10. $1038 / 326671 \mathrm{a} 0$

[45] Collett MJ, Loudon R. Popper versus Copenhagen. Nature 1987; 328 (6132): 675-676. http://dx. doi.org/10.1038/328675b0
[46] Shirai H. Reinterpretation of quantum mechanics based on the statistical interpretation. Foundations of Physics 1998; 28 (11): 1633-1662. http://dx . doi.org/10.1023/A:1018841625620

[47] Short AJ. Popper's experiment and conditional uncertainty relations. Foundations of Physics Letters 2001; 14 (3): 275-284. http://arxiv.org/abs/ quant-ph/0005063

[48] Short AJ. Momentum changes due to quantum localization. Fortschritte der Physik 2003; 51 (4-5): 498-503. http://arxiv.org/abs/ quant-ph/0112121

[49] Peres A. Karl Popper and the Copenhagen interpretation. Studies In History and Philosophy of Science Part B 1999; 33 (1): 23-34. http://arxiv.org/ abs/quant-ph/9910078

[50] Rigolin GG. Uncertainty Relations for Entangled States. Foundations of Physics Letters 2002; 15 (3): 293-298. http://arxiv.org/abs/ quant-ph/0008100

[51] Unnikrishnan CS. Popper's experiment, uncertainty principle, signal locality and momentum conservation. Foundations of Physics Letters 2000; 13 (2): 197-200. http://dx.doi.org/10.1023/A: 1007839718507

[52] Unnikrishnan CS. Is the quantum mechanical description of physical reality complete? Proposed resolution of the EPR puzzle. Foundations of Physics Letters 2002; 15 (1): 1-25. http://dx.doi.org/ 10.1023/A: 1015823125892

[53] Unnikrishnan CS. Proof of absence of spooky action at a distance in quantum correlations. Pramana 2002; 59 (2): 295-301. http://www.ias.ac.in/ pramana/aug2002/qt24.htm

[54] Popper KR. Objective Knowledge. London: Oxford University Press, 1972.

[55] Redhead M. Popper and the quantum theory. In: Karl Popper: Philosophy and Problems, O'Hear A (editor), Cambridge: Cambridge University Press, 1995, pp.163-176. http://dx.doi.org/ 10.1017/S1358246100005488 